\title{
AN A PRIORI ESTIMATE IN THE CALCULUS OF VARIATIONS
}

\author{
Steven C. Pinault
}

\begin{abstract}
This work is concerned with establishing an a priori estimate for the tilt excess of a $k$-dimensional varifold in $\mathbf{R}^{n}$ which is stationary with respect to the integral of a positive elliptic parametric integrand. For such a varifold, the tilt excess with respect to any $k$-plane $T$ is estimated a priori by the integral square deviation of the varifold from $T$. This estimate is applied in the author's $\mathrm{Ph}$. D. thesis, in the case of a $C^{2}$ two dimensional graph in $\mathbf{R}^{n}$ to derive an a priori pointwise bound on the slope.
\end{abstract}

1. Introduction. In the case of the area integrand, Allard [2] proved regularity for stationary varifolds with small integral deviation from a $k$-plane $T$. Regularity results for currents of small excess which minimize integrals of other elliptic integrands have been proven by Almgren [4] (and see Federer [5]) and Schoen and Simon [7]. Regularity results for a $C^{2}$ graph of codimension one stationary with respect to an elliptic integrand were proven by Simon [8].

In $\S 3$ of this work the estimate 8.13 of Allard [2] for the tilt excess of an area stationary varifold is extended to the case of integrands other than the area integrand. In the case that the codimension of the stationary varifold is greater than one the extension is only to those integrands which are $C^{1}$ close to the area integrand. In the case of codimension one, the estimate applies to any positive elliptic integrand.

In [6], we study $C^{2}$ two dimensional graphical submanifolds of $\mathbf{R}^{n}$ which are stationary with respect to an elliptic integrand $\Phi$ which is $C^{2}$ close to the area integrand. (In fact the graphical hypothesis can be replaced by the property that if $\xi$ is a 2 -vector field orienting the manifold, then $\xi$ omits a neighborhood of some simple vector in the unit sphere of $\Lambda_{2} \mathbf{R}^{n}$. For a graph the neighborhood is a hemisphere). The excess of such a manifold is estimated by its tilt excess, thus enabling us to approximate the manifold by a Lipschitz graph except for a set whose mass is estimated by the tilt excess. Using the fact that a $\Phi$-stationary two dimensional $C^{2}$ manifold has nonpositive Gauss curvature, (for $\Phi C^{2}$ close to the area integrand) so that its mass ratios are bounded from below [1], [6], the estimate of $\S 3$ then enables us to adapt the techniques of Theorems 8.16 and 8.19 of Allard [2] to obtain the regularity results for the manifold. 
2. Notation and definitions. Throughout this work we will use the notation of Allard [2] and Federer [5] unless otherwise indicated.

Whenever $0<r<\infty$ and $a \in \mathbf{R}^{n}$ we define

$$
U(a, r)=\left\{x \in \mathbf{R}^{n}:|x-a|<r\right\} .
$$

Let $\Phi$ be a positive elliptic parametric integrand of degree $n-1$ of $\mathbf{R}^{n}, \Phi: \mathbf{R}^{n} \rightarrow \mathbf{R}$. (See Chapter 5 of Federer [5].) We restrict our attention to those integrands which are even and have constant coefficients.

Let $G$ be an open subset of $\mathbf{R}^{n}$. We denote by $V_{n-1}(G)$ the set of $n-1$ dimensional varifolds on $G$, that is, the set of Radon measures on $G \times S^{n-1}$ which satisfy the condition $d V(x, w)=d V(x,-w)$ for $(x, w)$ $\in G \times S^{n-1}$ and $V \in V_{n-1}(G)$. We define $\|V\|(A)=V\left(A \times S^{n-1}\right)$ whenever $A$ is contained in $G$.

By Allard [3] we have the following formula for the first variation of $V$ with respect to the integral of $\Phi$ :

$$
\delta(V ; \Phi)(g)=\int_{G \times S^{n-1}} D g(x) \cdot\left(\Phi(w) 1_{\mathbf{R}^{n}}-D \Phi(w) w\right) d V(x, w)
$$

whenever $g: G \rightarrow G$ has compact support. We say $V$ is stationary with respect to $\Phi$ in $G$ if $\delta(V ; \Phi)(g)=0$ for all such $g$.

Throughout this work $c(\Phi)$ will be used to denote any constant depending only upon the quantities

$$
\sup \left\{\Phi(w),\|D \Phi(w)\|,\left\|D^{2} \Phi(w)\right\|: w \in S^{n-1}\right\}, \quad \inf \left\{\Phi(w): w \in S^{n-1}\right\},
$$

$n$, and the parametric Legendre condition bound for $\Phi$. (See Federer [5].)

We denote by $\Psi$ the area integrand, $\Psi(w)=|w|$.

3. The a priori estimate. Let $\Phi$ be as in $\S 2$ and suppose $U$ and $G$ are open subsets of $\mathbf{R}^{n}$. Let $0<\delta<\infty$ and suppose $U(x, \delta)$ is contained in $G$ whenever $x \in U$. Let $v \in S^{n-1}$. Then we have the following a priori estimate:

$$
\int_{U \times S^{n-1}}\left(1-(w \cdot v)^{2}\right) d V(x, w) \leq c(\Phi) \delta^{-2} \int_{G}(x \cdot v)^{2} d\|V\| x .
$$

Proof. Let $\phi: G \rightarrow \mathbf{R}$ be smooth with compact support. Then define $g(x)=\phi(x)^{2} x \cdot v \nabla \Phi(v)$. Since $V$ is stationary, we compute

$$
\begin{aligned}
& \int \phi(x)^{2}(\Phi(w) \Phi(v)-v \cdot \nabla \Phi(w) w \cdot \nabla \Phi(v)) \\
& \quad=-2 \int \phi(x) x \cdot v(\Phi(w) \nabla \Phi(v)-\nabla \Phi(v) \cdot w \nabla \Phi(w)) \cdot \nabla \phi(x) \\
& \quad \leq c(\Phi) \sup |\nabla \phi(x)| \int \phi(x)|x \cdot v||w-v| .
\end{aligned}
$$


Using Schwartz's inequality together with the inequality

$(*) \quad \Phi(v) \Phi(w)-w \cdot \nabla \Phi(v) v \cdot \nabla \Phi(w) \geq c(\Phi)\left(1-(v \cdot w)^{2}\right)$

(which follows from the convexity and evenness of $\Phi$ ) we obtain

$$
\int \phi(x)^{2}\left(1-(v \cdot w)^{2}\right) \leq c(\Phi) \sup |\nabla \phi(x)| \int(x \cdot v)^{2} .
$$

Choosing $\phi$ to be an appropriate cuttoff we obtain the stated result.

4. Extension to higher codimension. In the case of higher codimension the same result can be proven with the added hypothesis that $\Phi$ be close to the area integrand $\Psi$ in the sense that

$$
\sup \left\{|\nabla \Phi(\xi)-\xi|: \xi \in S_{k}\left(\mathbf{R}^{n}\right)\right\}
$$

be small (where we have used $S_{k}\left(\mathbf{R}^{n}\right)$ to denote the unit simple vectors in $\left.\Lambda_{k} \mathbf{R}^{n}\right)$.

To indicate where the extra hypothesis is needed, we define, for $w \in \mathbf{R}^{n}, Z_{\Phi}(w), L_{\Phi}(w): \mathbf{R}^{n} \rightarrow \mathbf{R}^{n}$ by

$$
L_{\Phi}(w)=\Phi(w) 1_{\mathbf{R}^{n}}-D \Phi(w) w, \quad Z_{\Phi}(w)=\Phi(w) 1_{\mathbf{R}^{n}}-L_{\Phi}(w) .
$$

With this notation we have

$$
\delta(V ; \Phi)(g)=\int D g(x) \cdot L_{\Phi}(w) d V(x, w),
$$

and the function $g$ of $\S 3$ is given by $g(x)=\phi(x)^{2} Z_{\Phi}(v)^{t}(x)$. The inequality $(*)$ of $\$ 3$ now takes the form

$$
L_{\Phi}(w) \cdot Z_{\Phi}(v)^{t} \geq c(\Phi)\left(1-(v \cdot w)^{2}\right)=c(\Phi) L_{\Psi}(w) \cdot Z_{\Psi}(v)^{t} .
$$

This inequality can be extended to the higher codimension case when $\Phi$ is close to $\Psi$ as above. For details see [6].

Acknowledgement. This work was completed at Duke University, where the author benefited greatly from the guidance and encouragement of William K. Allard.

\section{REFERENCES}

[1] H. Alexander and R. Osserman, Area bounds for various classes of surfaces, Amer. J. Math., 97 (1975), 753-769.

[2] W. K. Allard, On the first variation of a varifold, Ann. of Math., 95 (1972), 417-491.

[3] - On the first and second variation of a parametric integral, to appear.

[4] F. J. Almgren, Jr., Approximation of rectifiable currents by Lipschitz Q-valued functions, to appear. 
[5] H. Federer, Geometric Measure Theory, Springer-Verlag, New York, 1969.

[6] S. Pinault, An a priori estimate in the calculus of variations with an application to the regularity theory of nonlinear differential equations in two independent variables, Ph.D. Thesis, Duke University, 1981.

[7] R. Schoen and L. Simon, A new proof of the regularity theorem for rectifiable currents which minimize parametric elliptic functionals, Indiana Univ. Math. J., 1982.

[8] L. Simon, Equations of Mean Curvature Type in Two Independent Variables, Pacific J. Math., 69 (1977), 245-268.

Received June 14, 1982.

Western Electric Co.

Engineering Research Center

P. O. BoX 900

PRINCETON, NJ 08540 